\title{
Numerical results of two-dimensional $N=(2,2)$ super Yang-Mills theory
}

\section{Hidenori Fukaya, Issaku Kanamori*, Hiroshi Suzuki and Tomohisa Takimi}

Theoretical Physics Laboratory, RIKEN, 2-1 Hirosawa, Wako, Saitama 351-0198, Japan

E-mail: hfukaya@riken.jp, kanamori-i@riken.jp, hsuzuki@riken.jp,

ttakimi@riken.jp

We report the results of a numerical simulation of a lattice formulation of the two-dimensional $N=(2,2)$ super Yang-Mills theory proposed by Suzuki and Taniguchi [1]. We measure the 1point functions and 2-point functions. The scenario is that only tuning of the scalar mass to a specific value gives a supersymmetric continuum limit. Our results are consistent with this scenario although conclusive results on the restoration of supersymmetry have not been obtained.

The XXV International Symposium on Lattice Field Theory

July 30-4 August 2007

Regensburg, Germany

\footnotetext{
* Speaker.
} 


\section{Introduction}

A lattice formulation of the supersymmetric gauge theory is important to understand the nonperturbative aspects. Recently some formulations are proposed by several authors [2]. Most of the models use the fact that a nilpotent part of the supersymmetry can be kept on a lattice for $N \geq 2$ cases using the topological twist and the relations among some of them are now becoming transparent [3, 4, 5]. There is also an attempt to keep all the supersymmetry [6, 7]. Another approach is a model without exact supersymmetry [1] which uses the fact that in two-dimensional case, because of the super-renormalizability, there are only a few fine-tuning parameters to obtain the supersymmetric continuum limit. One of the merits of lattice formulation is to enable one to perform numerical simulations. In two-dimensional case, some results are known for the super Yang-Mills theory with the topological twist [8, 97.

In this talk, we report the result of a numerical simulation of the model without exact supersymmetry proposed in [1]. We measure some 1-point and 2-point functions.

\section{Model and Algorithm}

The target theory in the continuum is the 2-dimensional $N=(2,2)$ supersymmetric Yang-Mills theory which is obtained by a dimensional reduction from the 4-dimensional $N=1$ super YangMills. The lattice action is defined as a lattice version of a 4-dimensional action on $L \times L \times 1 \times 1$ lattice together with a scalar mass counter term $S_{\text {counter: }}{ }^{1}$

$$
S=S_{\mathrm{G}}+S_{\mathrm{F}}+S_{\text {counter }}
$$

The bosonic part is a plaquette action

$$
\begin{aligned}
S_{\mathrm{G}}[U] & =\frac{\beta}{2 N_{c}} \sum_{x \in \Gamma} \sum_{M, N} \operatorname{Retr}\{1-P(x, M, N)\}, \\
P(x, M, N) & =U(x, M) U(x+a \hat{M}, N) U(x+a \hat{N}, M)^{-1} U(x, N)^{-1},
\end{aligned}
$$

where we use $S U\left(N_{C}\right)$ gauge link variables, $U(x, \mu)=\exp \left(a g A_{\mu}^{a}(x) T^{a}\right),(\mu=0,1)$ and compact scalar fields, $U(x, 2)=\exp \left(\operatorname{ag} \varphi^{a}(x) T^{a}\right)$ and $U(x, 3)=\exp \left(\operatorname{ag} \phi^{a}(x) T^{a}\right)$. The coupling constant $g$ is related to $\beta$ through $\beta=2 N_{c} / a^{2} g^{2}$. The fermion action consists of the Wilson-Dirac operator

$$
S_{\mathrm{F}}[U, \lambda]=-a^{2} \sum_{x \in \Gamma} \operatorname{tr}\left\{\lambda(x) C D_{\mathrm{w}} \lambda(x)\right\}, \quad D_{\mathrm{w}}=\frac{1}{2} \sum_{M=0}^{3}\left\{\Gamma_{M}\left(\nabla_{M}^{*}+\nabla_{M}\right)-a \nabla_{M}^{*} \nabla_{M}\right\}
$$

with covariant differences for the adjoint representation $\nabla_{M}$

$$
\nabla_{M} \lambda(x)=\frac{1}{a}\left\{U(x, M) \lambda(x+a \hat{M}) U(x, M)^{-1}-\lambda(x)\right\}
$$

and its adjoint $\nabla_{M}^{*}$. The counter term is

$$
S_{\text {counter }}[U]=-\mathscr{C} N_{c} \sum_{x \in \Gamma}\left(\operatorname{tr}\left\{U(x, 3)+U(x, 3)^{-1}-2\right\}+\operatorname{tr}\left\{U(x, 2)+U(x, 2)^{-1}-2\right\}\right),
$$

\footnotetext{
${ }^{1}$ For the notational details, see [1].
} 
where $\mathscr{C}=0.65948255(8)$. The counter term is intended to cancel the radiative corrections to the scalar mass term. Other corrections which might appear in the effective action are suppressed in the continuum limit because of the super-renormalizability of this theory. All possible divergences in the sub-diagrams in the perturbative expansion are suppressed. It should be noted, however, that it does not guarantee the supersymmetry of composite operators.

In our numerical simulation, we use quenched gauge configurations generated by using the Hybrid Monte Carlo algorithm. The fermion contribution is introduced as a reweighting by the Pfaffian. In the continuum limit, the model has a real and positive Pfaffian. In fact the direct calculation for some sample configurations shows that the Pfaffian is real and positive in our parameter region. Therefore we use a positive square root of the determinant which numerical cost is much less expensive. We set a bare fermion mass $m=0$. The lattice size is $8 \times 8$ for 1-point functions and $12 \times 12$ for 2 -point functions. Since the coupling is $\beta=2 N_{c} / a^{2} g^{2}$, the continuum limit is the $\beta \rightarrow \infty$ limit. We set $3 \leq \beta \leq 40$. The gauge group is $S U(2)$.

We summarize the parameters and the numbers of independent configurations in table 1 .

\begin{tabular}{|c|c|c|c|c|c|c|c|c|c|}
\hline $\mathscr{C}$ & $\beta$ & 40 & 20 & 13 & 10 & 8 & 7 & 5 & 3 \\
\hline 0.001 & \multirow{6}{*}{$\begin{array}{c}\text { num. } \\
\text { of } \\
\text { configs. }\end{array}$} & 301 & 301 & 301 & 301 & 301 & 301 & - & - \\
\hline 0.10939 & & - & - & - & - & 301 & - & - & - \\
\hline 0.4 & & - & 9801 & - & - & 9801 & - & - & - \\
\hline 0.65948255 & & 801 & 9801 & 801 & 801 & 9801 & 801 & 801 & 801 \\
\hline 1.0 & & - & 9801 & - & - & 9801 & - & - & - \\
\hline 1.5 & & - & 9801 & - & - & 9801 & - & - & - \\
\hline \multicolumn{2}{|c|}{$a g$} & 0.316 & 0.447 & 0.555 & 0.632 & 0.707 & 0.756 & 0.894 & 1.154 \\
\hline
\end{tabular}

Table 1: The numbers of configurations for each parameter set on $8 \times 8$ lattice.

\section{One-point functions}

It is of our interest to measure the vacuum expectation value of a supercharge-exact operator, $\langle Q \mathscr{O}\rangle$, because it must vanish in the supersymmetric continuum limit. It should be noted, however, that $\langle Q \mathscr{O}\rangle$ can be non-zero (a finite renormalization), depending on the definition of the composite operator $Q \mathscr{O}$ which does not necessarily preserve the supersymmetry.

We make use of a scalar part of the topological twisted supercharges $Q$ and observe $Q$-exact 1point functions used in [8, 9]. Since we have no exact supersymmetry at finite lattice spacings, first we write down the continuum relations and then discretize them. We define the scalar supercharge $Q$ in the continuum as follows:

$$
\begin{array}{lll}
Q A_{\mu}^{a}=\psi_{\mu}^{a}, & Q \psi_{\mu}^{a}=i D_{\mu} \phi^{\prime a}, & Q \phi^{\prime a}=0, \\
Q \bar{\phi}^{\prime a}=\eta^{a}, & Q \frac{\eta^{a}}{2}=-\frac{i}{2} g f_{a b c} \phi^{\prime b} \bar{\phi}^{\prime c}, & Q \chi^{a}=i F_{01}^{a}
\end{array}
$$

Here we introduce scalar fields $\phi^{\prime}=\varphi+i \phi$ and $\bar{\phi}^{\prime}=\varphi-i \phi$. Fermions in the twisted basis $\psi_{\mu}, \eta$ and $\chi$ are given by liner combinations of the components of $\lambda:\left(\eta / 2, \chi, \psi_{0}, \psi_{1}\right)^{T} \equiv T \lambda$. 
We use the following three $\mathscr{O}_{i}$ 's:

$$
\mathscr{O}_{1}=-\frac{i}{8} g \eta^{a} f_{a b c} \phi^{\prime b} \bar{\phi}^{c}, \quad \mathscr{O}_{2}=-2 i \chi^{a} F_{01}^{a}, \quad \mathscr{O}_{3}=-\frac{i}{2} \psi_{\mu}^{a} D_{\mu} \bar{\phi}^{\prime a} .
$$

We divide $Q \mathscr{O}_{i}$ into two parts $Q \mathscr{O}_{i}=F_{i}+B_{i}$, where $F_{i}$ contains fermions and $B_{i}$ is made only from bosons:

$$
\begin{array}{lll}
F_{1}=-\frac{i}{8} g f_{a b c} \phi^{\prime a} \eta^{b} \eta^{c}, & F_{2}=i \chi^{a}\left(D_{0} \psi_{1}^{a}-D_{1} \psi_{0}^{a}\right), & F_{3}=\frac{i}{2} \psi_{\mu}^{a} D_{\mu} \eta^{a}+\frac{i}{2} g f_{a b c} \bar{\phi}^{\prime a} \psi_{\mu}^{b} \psi_{\mu}^{c}, \\
B_{1}=-\frac{1}{8} g^{2}\left(f_{a b c} \phi^{\prime b} \bar{\phi}^{\prime c}\right)^{2}, & B_{2}=2\left(F_{01}^{a}\right)^{2}, & B_{3}=\frac{1}{2} D_{\mu} \phi^{\prime a} D_{\mu} \bar{\phi}^{\prime a} .
\end{array}
$$

In the continuum, the kinetic terms and Yukawa interactions of the twisted fermions are contained in the Dirac operator. Therefore, to find an appropriate discretization of these terms, we simply replace the continuum Dirac operator with the Wilson-Dirac operator. For example, we replace

$$
i \psi_{0} D_{0} \frac{\eta}{2} \Rightarrow \psi_{0}\left(\left(T^{-1}\right)^{T}\left(C D_{\mathrm{W}}\right) T^{-1}\right)_{\psi_{0}} \eta \frac{\eta}{2} .
$$

All dimensionful observables are measured in a unit of the dimensionful coupling $g$.

First, we present the result of the $\mathscr{O}_{1}$ case. Figure 1 shows that each of the bosonic and fermionic parts is divergent in the continuum limit. The sum $\left\langle Q \mathscr{O}_{1}\right\rangle$ stays finite after the reweighting by the Pfaffian, while the quenched result diverges (Fig. 2). In this theory, the cancellation of divergences in $\left\langle Q \mathscr{O}_{1}\right\rangle$ is achieved by a balance between bosons' and fermions' degrees of freedom. Our reweighted result is consistent with this fact and the effect of dynamical fermions appears to be properly included by the reweighting. As already noted, even in the supersymmetric continuum limit, $\left\langle Q \mathscr{O}_{1}\right\rangle$ can be non-zero due to a finite renormalization. The $\mathscr{C}$ dependence of $\left\langle Q \mathscr{O}_{1}\right\rangle$ is summarized in Figure 3. To estimate the effect of the finite renormalization, we determined the values of $\mathscr{C}$ which provide $\left\langle Q \mathscr{O}_{1}\right\rangle=0$. They are $\mathscr{C}=1.047(51)$ at $\beta=8$ and $\mathscr{C}=1.006(77)$ at $\beta=20$. Almost no $\beta$-dependence is observed. These values are significantly different from $\mathscr{C}=0.65948255$ calculated in the continuum limit [1] ] and suggest that the effect of the finite renormalization is certainly not negligible.

The cancellation of divergences is also realized both in $Q \mathscr{O}_{2}$ and $Q \mathscr{O}_{3}$ cases (Fig. (4). The $\mathscr{C}$-dependence, however, is not manifest. Note that the plots have much more errors than that $\left\langle Q \mathscr{O}_{1}\right\rangle$ and this would imply that the $\mathscr{C}$-dependence is smeared. The difference between behavior of $Q \mathscr{O}_{1}$ and that of $Q \mathscr{O}_{2}$ and $Q \mathscr{O}_{3}$ could be accounted as a result of the difference of the divergence of each operators. $B_{1}$ and $F_{1}$ have logarithmic divergences, while $B_{2}, B_{3}, F_{2}$ and $F_{3}$ have quadratic divergences.

\section{Two-point functions}

Next we measure quantities with which the restoration of supersymmetry is expected to be observed transparently. A supersymmetric Ward-Takahashi identity indicates that the following 2-point functions should have the same functional form: ${ }^{2}$

$$
B=2 i\left\langle j_{5 \mu}(x) j_{v}(y)\right\rangle, \quad F=\left\langle\operatorname{tr}\left\{\gamma_{\mu} \gamma_{5}\left(\phi^{a}+i \gamma_{5} \varphi^{a}\right) \psi^{a}(x) j_{v}^{\text {super }}(y)\right\}\right\rangle,
$$

\footnotetext{
${ }^{2}$ The former function $B$ exhibits a power law behavior in the continuum theory [10].
} 

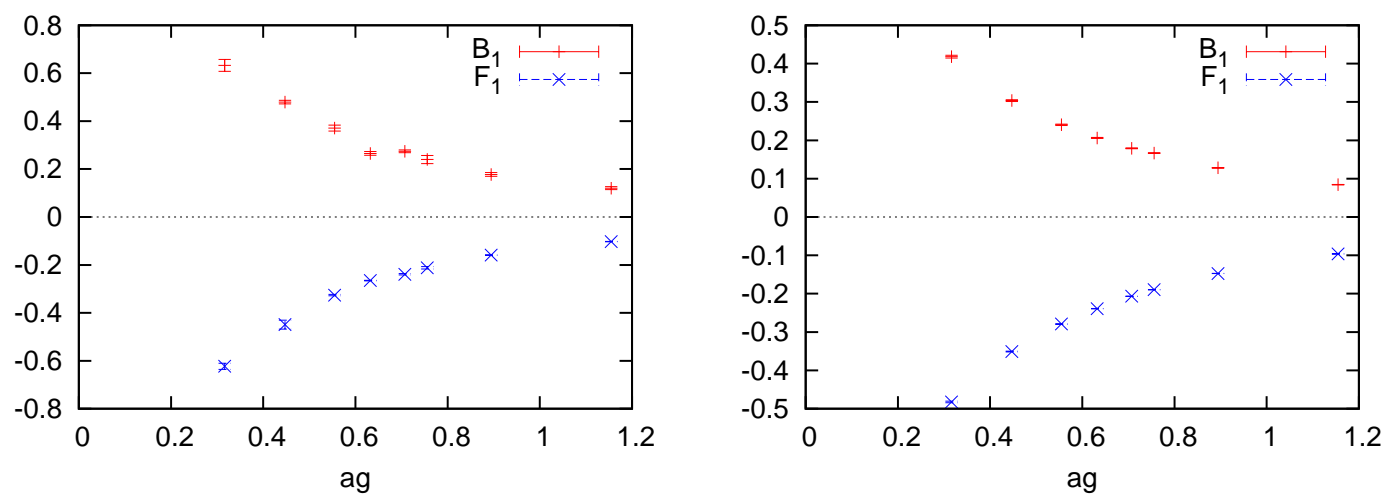

Figure 1: The $a \rightarrow 0$ limit of $B_{1}$ and $F_{1}$, the left is reweighted and the right is quenched. The counter term is $\mathscr{C}=0.65948255$. The lattice size is $8 \times 8$.
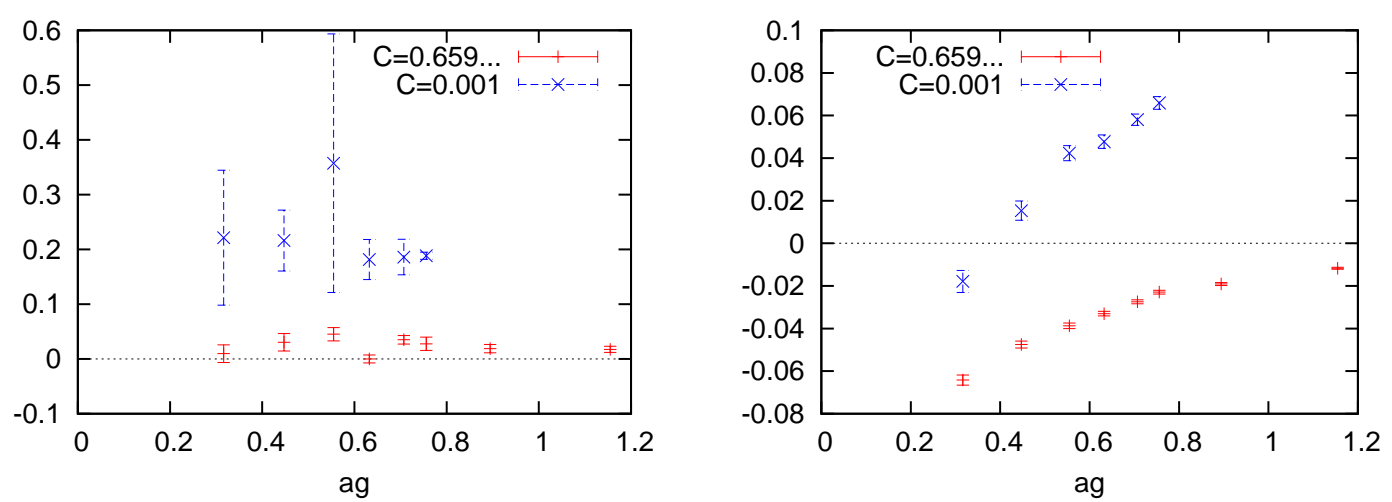

Figure 2: The $a \rightarrow 0$ limit of $\left\langle Q O_{1}\right\rangle$, the left is reweighted and the right is quenched. The lattice size is $8 \times 8$.

where the bosonic currents are

$$
j_{\mu}(x)=\bar{\psi}^{a} \gamma_{\mu} \psi^{a}(x), \quad j_{5 \mu}(x)=\bar{\psi}^{a} \gamma_{\mu} \gamma_{5} \psi^{a}(x)+2 i\left\{\phi^{a} \partial_{\mu} \varphi^{a}(x)-\varphi^{a} \partial_{\mu} \phi^{a}(x)\right\}
$$

and the fermionic current is

$$
j_{\mu}^{\text {super }}(x)=\bar{\psi}^{a} \gamma_{\mu}\left\{\frac{1}{2} F_{\rho \sigma}^{a} \sigma_{\rho \sigma}-i \gamma_{\rho} D_{\rho}\left(\phi^{a}+i \gamma_{5} \varphi^{a}\right)-i g f_{a b c} \varphi^{b} \phi^{c} \gamma_{5}\right\}(x) \text {. }
$$

Figure 5 shows a typical result of the 2-point functions. The number of the configurations we used is 101 and the bare fermion mass is 0 . According to the scenario, we expect that a suitable choice of $\mathscr{C}$ should give the supersymmetric result, i.e., the identical spectra, while the other choices of $\mathscr{C}$ should not. Unfortunately, errors in the plot are too large to analyze the spectra although this is a result with the quenched approximation. The point here is that we cannot distinguish the difference of the counter term.

\section{Conclusion}

We observed 1-point functions and 2-point functions in a lattice formulation of the twodimensional $N=(2,2)$ super Yang-Mills theory. In our scenario, only the counter term coefficient 

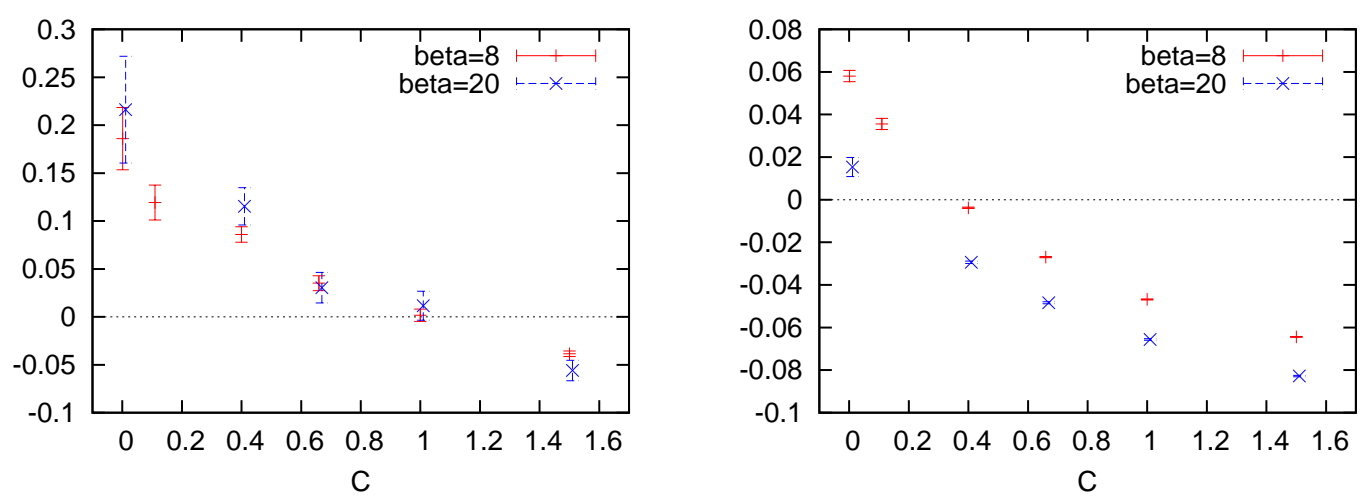

Figure 3: The $\mathscr{C}$ dependence of $\left\langle Q O_{1}\right\rangle$, the left is reweighted and the right is quenched. The lattice size is $8 \times 8$.
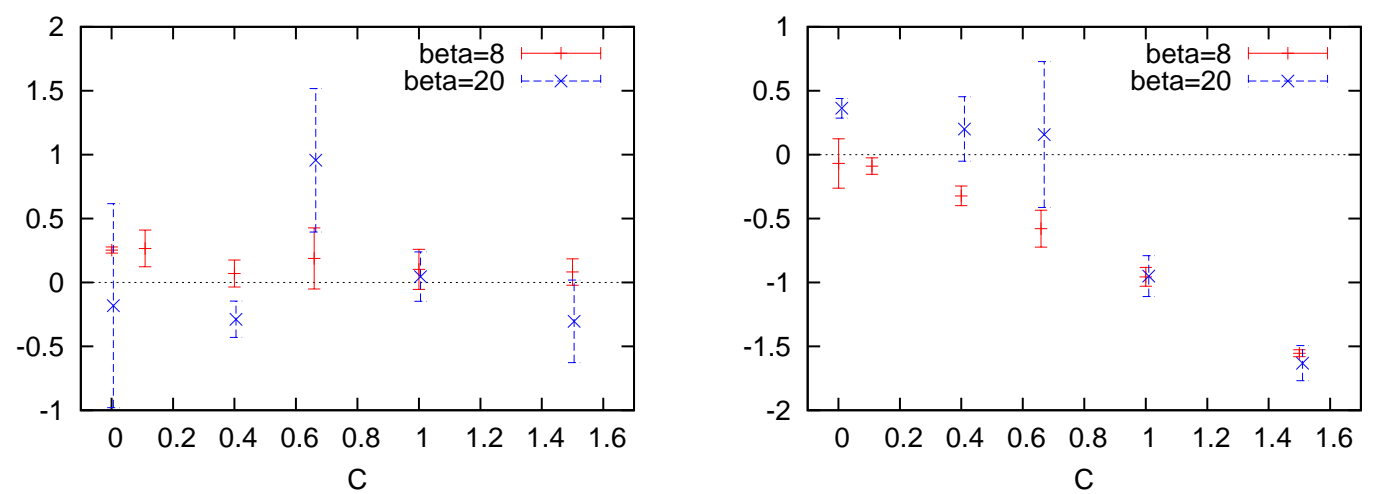

Figure 4: The $\mathscr{C}$ dependence of $\left\langle Q O_{2}\right\rangle$ (left) and $\left\langle Q O_{3}\right\rangle$ (right). These are reweighted and the lattice size is $8 \times 8$.

$\mathscr{C}$ should be finely tuned. The 2-point functions have large errors that we cannot compare the spectra associated with a bosonic current and a fermionic current. The 1-point functions we observed are finite in the continuum limit because of the fermion loop effect. The result of a less divergent 1-point function depends on $\mathscr{C}$ and is consistent with our scenario. To obtain the conclusive result from this dependence, i.e., whether the scenario actually works or not, we need the renormalization factor for the 1-point function. Although the current result is not quite promising, we have some possible ways to improve. A UV-filtered reweighting will help to reduce the errors after the reweighting. The HMC algorithm with dynamical fermions is another option. The result of 2-point functions suggests that the fermion or the scalar (or both) is rather far from massless so that a negative bare mass of the fermion which reduces the physical mass may improve the sensitivity on the counter term.

\section{Acknowledgments}

We would like to thank Yusuke Taniguchi for discussion at the early stage of this work. We thank for computational resources of the RIKEN Super Combined Cluster (RSCC). I.K. is supported by the Special Postdoctoral Researchers Program at RIKEN. The work is supported in part by Grant-in-Aid for Scientific Research, Nos. 18840045 (H.F.) and 18540305 (H.S.), and by 

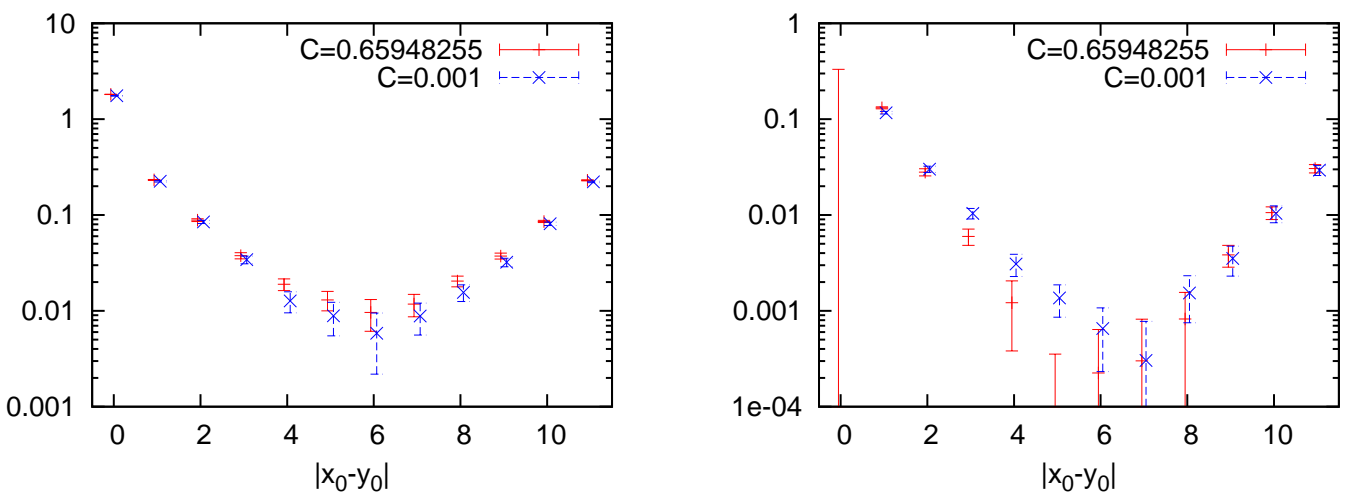

Figure 5: The two-point functions, $B$ (left) and $F$ (right), in the quenched approximation. These are $\mu=1$, $v=0$ components of eq. 4.1 . The parameters are $\beta=18$ and $\mathscr{C}=0.65948255,0.001$ on $12 \times 12$ lattice.

JSPS and French Ministry of Foreign Affairs under the Japan-France Integrated Action Program (SAKURA).

\section{References}

[1] H. Suzuki and Y. Taniguchi, Two-dimensional $N=(2,2)$ super Yang-Mills theory on the lattice via dimensional reduction, JHEP 10 (2005) 082, hep-lat/0507019.

[2] J. Giedt, Advances and applications of lattice supersymmetry, PoS LAT2006 (2006) 008, hep-lat/0701006], and references therein.

[3] T. Takimi, Relationship between various supersymmetric lattice models, JHEP 07 (2007) 010, [0705.3831.

[4] P. H. Damgaard and S. Matsuura, Relations among supersymmetric lattice gauge theories via orbifolding, 0706.3007.

[5] P. H. Damgaard and S. Matsuura, Classification of supersymmetric lattice gauge theories by orbifolding, JHEP 07 (2007) 051, 0704.2696.

[6] A. D'Adda, I. Kanamori, N. Kawamoto, and K. Nagata, Exact extended supersymmetry on a lattice: Twisted $N=2$ super Yang-Mills in two dimensions, Phys. Lett. B633 (2006) 645-652, [hep-lat/0507029].

[7] A. D'Adda, I. Kanamori, N. Kawamoto, and K. Nagata, Exact extended supersymmetry on a lattice: Twisted $N=4$ super Yang-Mills in three dimensions, 0707.3533 .

[8] S. Catterall, On the restoration of supersymmetry in twisted two- dimensional lattice Yang-Mills theory, JHEP 04 (2007) 015, hep-lat/0612008].

[9] H. Suzuki, Two-dimensional $\mathscr{N}=(2,2)$ super Yang-Mills theory on computer, 0706.1392 .

[10] H. Fukaya, I. Kanamori, H. Suzuki, M. Hayakawa, and T. Takimi, Note on massless bosonic states in two-dimensional field theories, Prog. Theor. Phys. 116 (2007) 1117-1129, hep-th/ 0609049. 